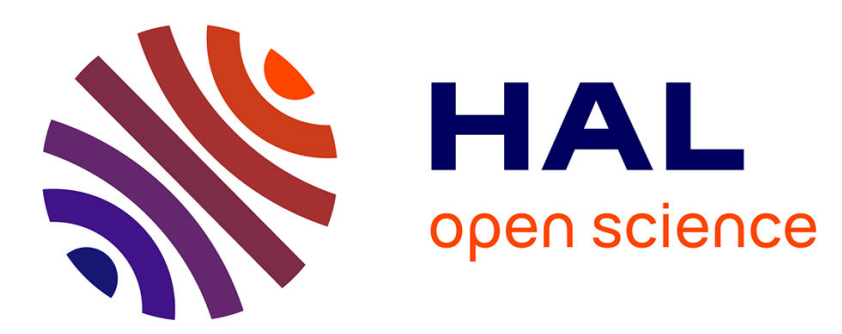

\title{
Measuring bow force in bowed string performance: Theory and implementation of a bow force sensor
}

\author{
Matthias Demoucron, Anders Askenfelt, René Causse
}

\section{To cite this version:}

Matthias Demoucron, Anders Askenfelt, René Causse. Measuring bow force in bowed string performance: Theory and implementation of a bow force sensor. Acta Acustica united with Acustica, 2009, 95 (4), pp.718-732. 10.3813/AAA.918200 . hal-00444903

\section{HAL Id: hal-00444903 https://hal.science/hal-00444903}

Submitted on 7 Jan 2010

HAL is a multi-disciplinary open access archive for the deposit and dissemination of scientific research documents, whether they are published or not. The documents may come from teaching and research institutions in France or abroad, or from public or private research centers.
L'archive ouverte pluridisciplinaire HAL, est destinée au dépôt et à la diffusion de documents scientifiques de niveau recherche, publiés ou non, émanant des établissements d'enseignement et de recherche français ou étrangers, des laboratoires publics ou privés. 


\title{
Measuring bow force in bowed string performance: Theory and implementation of a bow force sensor
}

\author{
M. Demoucron*, A. Askenfelt ${ }^{\dagger}$ and R. Caussé* \\ *IRCAM, Centre Pompidou, CNRS UMR 9912 \\ 1 Place Igor Stravinsky, 75004 Paris, France \\ *KTH-Computer Science and Communication, \\ Dept. of Speech Music and Hearing \\ Lindstedtsvägen 24, SE-100 44 Stockholm, Sweden
}

\begin{abstract}
A sensor has been developed which allows measurement of the force exerted by the bow on the string (bow force) during violin performance. The bow force is deduced from measurement of the transversal force at the termination of the bow hair at the frog. The principle is illustrated with an experiment that demonstrates how the bending of the stick and variations in bow hair tension influence the measurements. The design of the sensor is described and performance characteristics are discussed. A thorough calibration procedure is described and tested. Finally, the use of the sensor is demonstrated through measurements in real playing situations.
\end{abstract}

\section{INTRODUCTION}

Bow force is one of the three main control parameters in bowed string playing, the two others being bow velocity and bowbridge distance. In particular, a rapid establishment of Helmholtz motion during the attack is dependent on the coordination of bow force with acceleration and bow bridge distance [6][11]. Also, for the control of timbre, bow force is the main parameter, bow-bridge distance playing a secondary role [7].

The interest for measuring control parameters in playing is encouraged by the advancements in the study of musical instruments by physical modeling. One advantage of this approach is that the control parameters of the model become the same as for the real instrument. On the other hand, measuring the control parameters in normal playing without interfering with normal playing conditions presents a challenge, in particular for wind instruments. The bowed stringed instruments are seemingly straight-forward to approach as the string and bow are of reasonable size and the motions associated with bowing are accessible for measurement. Application of motion capture techniques is an obvious approach for measuring bow velocity and bow-bridge distance in playing, while bow force measurements will require application of sensors to the bow or the violin.

The first reliable measurement of control parameters in violin playing during real performance were made by Askenfelt [1], [2]. A bow was modified to measure bow position, bow velocity, bow-bridge distance and bow force. Bow position refers to the position of the contact point between bow hair and string relative to the frog or tip. The bow position was measured by a resistance wire running among the hairs in the outer layer of the bundle of bow hair contacting the string. The string divided the wire in two parts which were used in a branch of a Wheatstone bridge. Bow-bridge distance was measured in a similar manner, using the parts of the string on each side of the bow (wire) in a second Wheatstone bridge. Bow velocity was obtained from the bow position signal through differentiation. Bow force was measured by gluing the bow hair to two metal strips at the frog and at the tip, respectively, and measuring the deflection of the strips by strain gauges.

Later, Young used the bending of the bow stick to estimate the force on the string in playing. Two pairs of strain gauges were glued to the stick in order to measure the bow force (normal to the string) as well as the lateral force component (in the string direction) [13]. Recently, the present author [4] proposed an improvement of Askenfelt's work by designing detachable sensors which could be attached to any bow, and treating the sensor signals from the frog and tip independently. Later implementations of this idea for real-time measurements have been presented by Guaus et al [5]. The following presentation gives a description of the basic idea, theory, design, and calibration of a detachable bow force sensor. 


\section{INTRODUCTORY EXPERIMENT AND THEORETICAL CONSIDERATIONS}

The basic idea of the force sensor was to determine bow force indirectly by measuring the resulting transversal forces at the bow hair terminations. In this section, the principle is illustrated and the influence of the mechanical behaviour of the bow is analysed. A simple model based on measurements is used to quantify the contributions from the bending of the bow stick and changes in bow hair tension to the observed behaviour.

\section{A. General description of the bow}

The bow consists of a bow hair ribbon fastened to the head of the bow stick at one end and to the frog at the other end (see Fig. 1a). As the bow hair is brought up to tension by turning the frog screw, the hair pulls the head back, and the initial camber of the stick is reduced. Keeping the frog fixed horizontally, the tip is lowered until an initial displacement $h_{t}^{0}$ has been reached, at which the restoring force resulting from the straightening of the stick counteracts the static hair tension (see Fig. 1b). The displacement of the tip is measured relative to a horizontal reference line extending from the underside of the frog, as if the frog was resting on a table (see Fig. 1). ${ }^{1}$

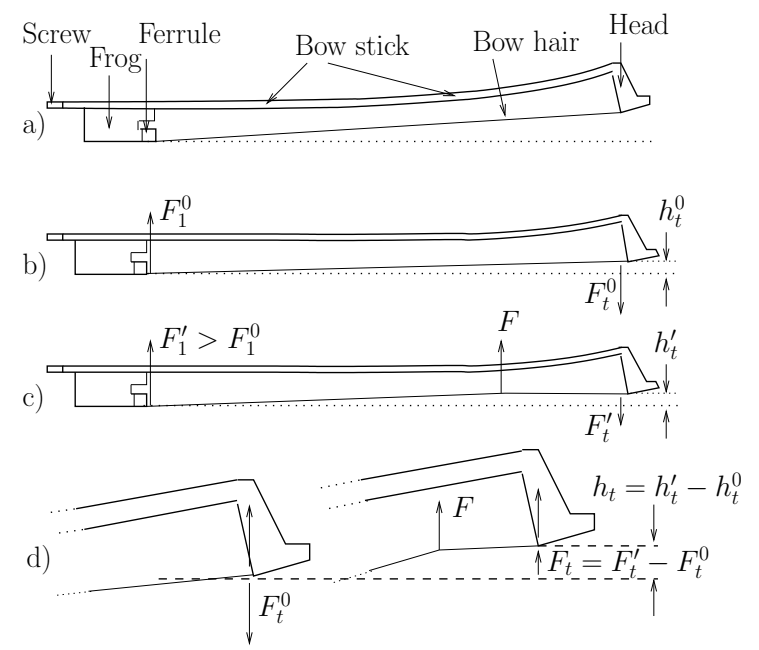

Figure 1. Description of the bow geometry and definition of forces and displacements: (a) Bow hair not tightened, (b) bow hair tightened, (c) bow force $F$ applied at the bow hair. $F_{1}^{\prime}$ represents the transversal force acting at the frog, and $-F_{t}^{\prime}$, the transversal force acting at the tip. The reaction of the bow-stick resulting from the straightening is indicated by $F_{t}^{\prime}$.

When the bow is pressed against the string, the bow hair is deflected at the contact point, resulting in a bow force $F$ normal to the string. Associated transversal reaction forces act at the frog $F_{1}$, and at the tip $F_{t}$, respectively (see Fig. 1c). At the frog, the change in force is visible through the change in bow hair angle relative to the reference line, and at the opposite end through changes in the displacement of the tip $h_{t}^{\prime}$ (see Fig. 1d). The deflection of the bow hair due to the applied bow force relaxes the transversal force on the tip $\left(F_{t}^{\prime}<F_{t}^{0}\right)$, because the slope of the bow hair at the tip will be directed more upwards. As a result, the bow stick regains some of the initial camber. Simultaneously, the bow hair tension will tend to increase.

In the following, $T_{0}$ will be used to indicate the initial tension set by the player, and $T$ the effective tension that may vary when applying the bow force. Normalized bow position is denoted by $\gamma$, indicating the ratio of the distance $x_{b}$ between the frog and the contact point with the string (bow position), and the length of the bow hair $L_{b}(0<\gamma<1)$. All variables will be expressed relative to the configuration in which the bow hair is tightened and no bow force applied

$$
F_{1}=F_{1}^{\prime}-F_{1}^{0} \quad, \quad F_{t}=F_{t}^{\prime}-F_{t}^{0} \quad, \quad h_{t}=h_{t}^{\prime}-h_{t}^{0}
$$

\footnotetext{
${ }^{1}$ Superscript 0 will be used to denote the condition with the bow hair at playing tension and no bow force applied, and prime symbols to indicate playing condition with bow force applied.
} 


\section{B. Experiment}

A test bench was designed for the initial experiments. With the bow hair tightened, the bow was rigidly clamped upside-down at the frog, with the tip being free to move. A force transducer (HBM model U1A, compliance $0.028 \mathrm{~mm} / \mathrm{N}$, frequency range 0 - ca $200 \mathrm{~Hz}$ ) was used to measure the transversal force component $F_{1}$ of the hair close to the frog. This was done by letting the bow hair run on top of a small wheel, mounted on the facing of the transducer (see Fig. 2). At the free end of the bow, a digital caliper was used to measure the tip displacement $h_{t}$. From repeated measurements the accuracy was estimated to \pm 0.2 $\mathrm{mm}$.

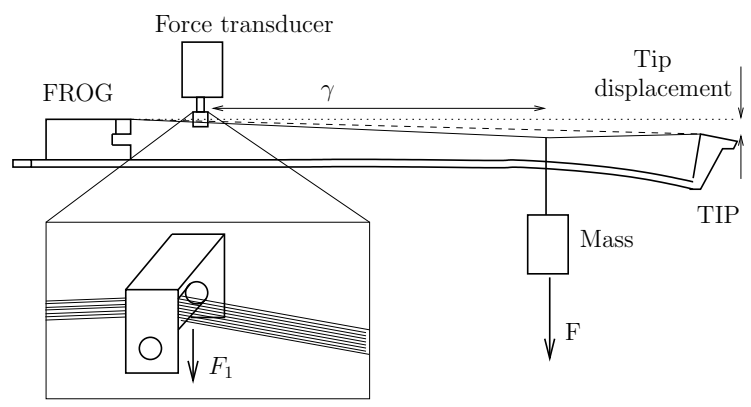

Figure 2. View of the experiment for determining transversal forces at the bow hair terminations. The bow is clamped upside-down at the frog. Bow forces were simulated by hanging masses on the bow hair. The resulting transversal forces are measured by a force transducer close to the frog and by the displacement of the tip.

Loads, simulating different bow forces $F$, were applied by hanging weights with known mass at six positions distributed across the entire length of the bow hair. Three masses were used, 54, 100 and $154 \mathrm{~g}$ (approximately 0.5, 1, and 1.5 N). The values were chosen to cover the typical bow force range in normal playing, from 0.5 to $1.5 \mathrm{~N}$ [1]. Each measurement series was repeated three times.

\section{Results}

1) Force at the tip: Tip displacements for the three loads are shown in Fig. 3a. Each load case includes three curves corresponding to the repeated measurements. For a given load the tip displacement increases with bow position $\gamma$, and for a given position, with increasing load.
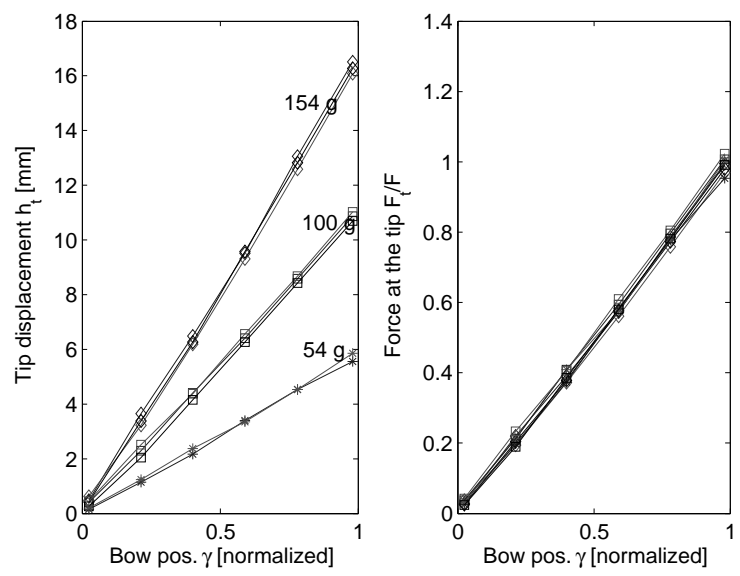

Figure 3. (a) Measured tip displacement vs. normalized position for a load on the bow hair (54, 100 and $154 \mathrm{~g}$ ), equivalent to applying bow forces of 0.5 , 1 , and $1.5 \mathrm{~N}$ at different bow positions $\gamma$. Each series was repeated three times. (b) Calculated transversal force at the tip $F_{t}$ normalized by bow force $F$ from equation 2 using $K_{b}=91 \mathrm{~N} / \mathrm{m}$.

The displacements are due to the transversal force acting at the tip when the bow hair is loaded. The data in the figure indicate that the assembled, tightened, bow can be well described by a transverse stiffness $K_{b}$. The force acting on the tip can 
then be calculated from the tip displacement

$$
F_{t}=K_{b} h_{t}
$$

An estimation of the transverse stiffness $K_{b}$ was obtained by taking the average of the three tip displacements at the tip $(\gamma=1)$, giving a value of $91 \mathrm{~N} / \mathrm{m}$. The resulting force at the tip $F_{t}$ for relative bow positions was computed using this value and equation 2 (see Fig. 3b). The force values have been normalized relative to the bow force $F$. As seen, the normalized force at the tip can be well approximated by a linear increase from 0 to 1 as function of $\gamma$

$$
F_{t}=\gamma F
$$

2) Force at the frog: The force measurements from the transducer at the frog vs. normalized load position are shown in Fig. 4. The data points of the three repeated measurements merge, indicating a high reproducability in the experiment.

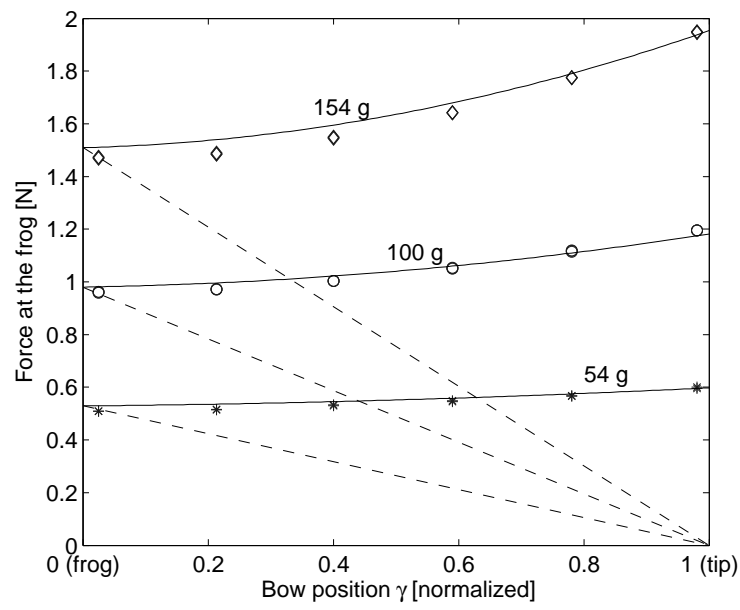

Figure 4. Transversal force at the frog $F_{1}$ vs. position of a load on the bow hair $(54,100$ and $154 \mathrm{~g}$ ), equivalent to applying bow forces of $0.5,1$, and 1.5 $\mathrm{N}$ at different bow positions $\gamma$. The forces measured by the transducer close to the frog are indicated by data points. The dashed lines represent a simplified bow model with a completely rigid stick. The full lines correspond to a realistic model (equation 11), accounting for tip displacement and variations in bow hair tension. Data were fitted with $T_{0}=50.2 \mathrm{~N}$ and $\alpha_{T}=8.1 \mathrm{~N} / \mathrm{N}$.

The force at the frog $F_{1}$ is seen to increase with increasing load position (moving away from the frog), which may seem surprising. Intuitively the expected behaviour would be that the force gets lower as the tip is approached, due to the decreasing angle of the bow hair at the frog. With the mass placed at the transducer $(\gamma=0)$, the measured force would correspond to the applied weight, and as the loading point moves closer to the tip, the force at the frog would approach zero. The simplified case in which the bow stick is completely rigid gives

$$
F_{1}=(1-\gamma) F
$$

The results obtained with such a rigid model are included in Fig. 4 (straight dashed lines). The measurements are far from following this simple behaviour. In fact the measured force at the frog increases substantially when the load is moved farther away, and surprisingly the force at the frog is greater with the load at the tip than at the frog. In the following section two factors that explain this behaviour, tip displacement and bow hair tension, are analyzed.

\section{Analysis}

In the following analysis, a simple model is used to represent the bow. The assembled bow when tightened is supposed to act as a spring in the transverse direction, with stiffness $K_{b}$ at the tip. The bow hair ribbon is represented by a single string with tension $T$ and length $L_{b}$. The displacement of the hair at the bow position is denoted by $h(\gamma)$. The transversal force at the frog can then be written as

$$
F_{1}=T \frac{h(\gamma)}{\gamma L_{b}}
$$


1) Tip displacement: A study including the bending of the stick has been presented by Pitteroff [8], aiming at a description of the transverse stiffness along the bow hair. He considered that the force acting at the tip was $\gamma F$, which is in line with our measurements (see Fig. 3).

Using this assumption, Pitteroff found the displacement of the hair at the bowing point $\gamma$ to be composed of two terms, the first one representing the deflection of bow hair under loading when the bow tip does not move, and the second one taking the global displacement of bow hair due to the tip displacement $h_{t}$ (equation 2) into account

$$
h(\gamma)=\frac{\gamma(1-\gamma) L_{b}}{T} F+\frac{\gamma^{2}}{K_{b}} F
$$

The force acting at the frog is obtained from equations 6 and 5

$$
F_{1}=\left[1-\gamma\left(1-\frac{T}{K_{b} L_{b}}\right)\right] F
$$

The effect of stick bending reduces the slope of the dashed line in Fig. 4 representing the transversal force at the frog, and the force does not reach zero at the tip, as was the case with the rigid model. The reason is that when the contact point is moved towards the tip, the decrease in angle of the bow hair at the frog is more than compensated by the bending of the bow, which lowers the position of the hair termination at the tip.

The stick bending can dominate the displacement of the bow hair at the contact point and give an increasing force at the frog when approaching the tip. From equation 7, this will be the case if the tension is greater than $K_{b} L_{b}$

$$
T \geq K_{b} L_{b} \Rightarrow \frac{\partial F_{1}}{\partial \gamma} \geq 0
$$

In our case with $K_{b}=91 \mathrm{~N} / \mathrm{m}$ and $L_{b}=0.53 \mathrm{~m}$ this will occur when the bow hair tension exceeds $48 \mathrm{~N}$. For comparison, Askenfelt [3] and Pitteroff [8] reported typical tensions to be around $60 \mathrm{~N}$, with an estimated practical lower limit of $45 \mathrm{~N}$ which was considered "very loose" but still playable. The conditions for a considerable influence of the bending of the stick are thus at hand with the bow hair taught to normal playing conditions.

The theoretical behaviour of the transversal force at the frog partly matches the observed data in Fig. 4, explaining the observed increase in force with increasing $\gamma$. The model does not, however, succeed in explaining the non-linear variation with $\gamma$. It is therefore necessary to examine the influence of the hair tension $T$ in equation 7 , and how it can contribute to a complete explanation of the observed data.

2) Variation in bow hair tension: Accurate measurement of bow hair tension for the assembled bow is not straight-forward to perform without instrumenting the bow. In studies of the bow, the tension is generally described as "low", "normal" or "high". Askenfelt [3] reported a simple estimation of acceptable bow hair tensions using a dynamometer. The player was first asked to tighten the bow with the screw, the position of the frog on the bow stick was then measured, and, the screw being removed, the frog was then pulled to the same position with a dynamometer allowing an estimation of the hair tension. More accurate estimations can be made by clamping the bow rigidly at the frog and tip and measure the (small) deflection of the bow hair when loaded at the middle by a suitable weight. By making the measuring point electrically conductive by a small piece of cupper tape to indicate in-contact condition, an accuracy of $0.05 \mathrm{~mm}$ can be reached in the measurements using a dial gauge.

In this study, we took advantage of the unused part of the bow hair between the frog and the force transducer. A second force transducer (transducer 2) was placed in the middle of the unused part, pressing slightly on the bow hair (see Fig. 5). Provided that the facing of transducer 1 is rigid, the signal from transducer 2 is sensitive only to variations $\delta T$ in the static bow hair tension $T_{0}$

$$
F_{2} \propto T_{0}+\delta T
$$

In the experiment, the support at transducer 1 approximated a rigid termination well with a nominal displacement of the facing of $0.028 \mathrm{~mm} / \mathrm{N}$. 


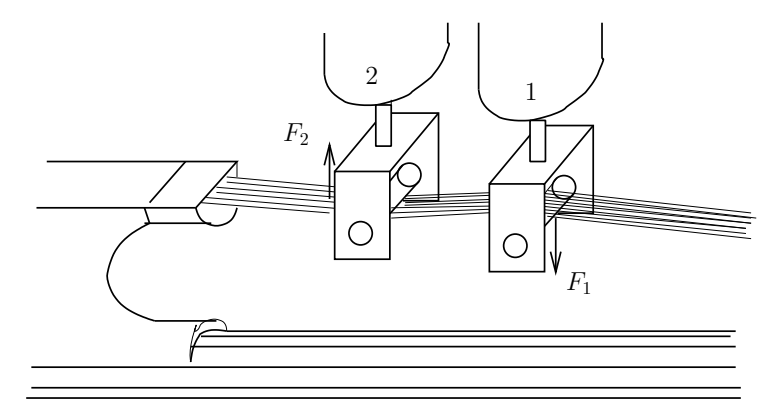

Figure 5. Two force transducers were used to measure the variation in bow hair tension during loading of the bow hair. Provided that the facing of transducer 1 is rigid, transducer 2 is sensitive to tension variations only.

The setup was used to quantify variations in bow hair tension when loading the bow. It was not possible to make an absolute calibration of the force variations with the assembled bow, but the ratio between the variations and the static signal with no loading applied gave the relative tension variation. The results are plotted versus bow position for the three loads (see Fig. 6, left).

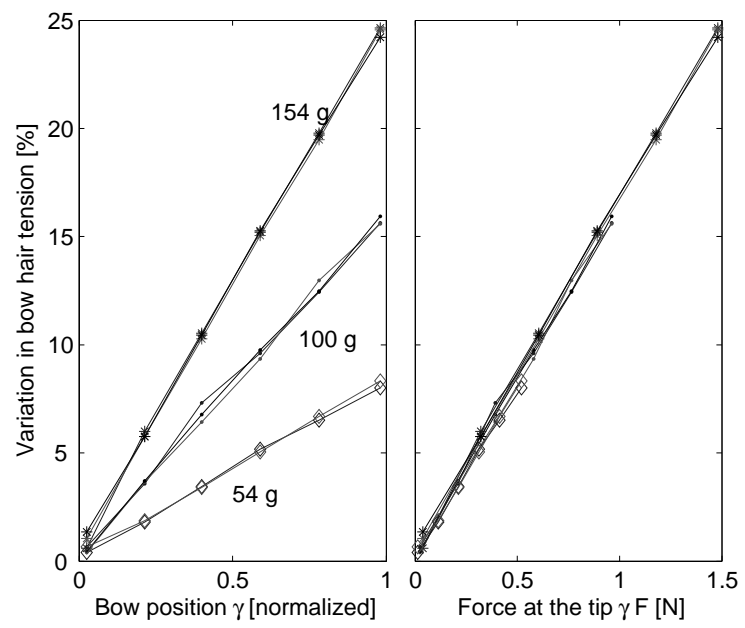

Figure 6. Left: Variation in bow hair tension vs. normalized position $\gamma$ of a load on the bow hair (54, 100 and $154 \mathrm{~g})$. Right: The same variations in bow hair tension vs the calculated transversal force at the tip $F_{t}=\gamma F$.

The curves corresponding to the different loads are seen to be well separated and almost linear. The variations in bow hair tension during playing are often assumed to be rather small, but in the present case they reached $25 \%$ of the initial tension for the highest load (154 g) at the tip.

In the following, it is assumed that the variations in hair tension are mainly related to the tip displacement, neglecting the elongation of the hair under loading. An experiment, in which the stick was prevented from moving at the tip, was made in order to confirm this assumption. The tension variation was then less than $1 \%$ with $1 \mathrm{~N}$ load at the middle of the hair, but increased to around $10 \%$ when the bow stick was released at the tip and free to bend. As a good approximation, the tension variation due to the deflection of the bow hair under loading can be considered not significant compared to the variation due to tip displacement. Tension variation can then be expressed as a function of the transversal force at the tip $F_{t}=\gamma F$. As seen in Fig. 6 (right), the variation in tension can be approximated by a linear relation

$$
T=T_{0}+\alpha_{T} \gamma F
$$

where $\alpha_{T}$ is a coefficient quantifying the combined effect of bow force and bow position. 
With the use of Eq. 10, equation 7 becomes

$$
F_{1}=\gamma^{2} \frac{\alpha_{T}}{K_{b} L_{b}} F^{2}+\left(1-\gamma+\gamma \frac{T_{0}}{K_{b} L_{b}}\right) F
$$

The ratio between $\alpha_{t}$ and $T_{0}$ was found to be $0.16 \%$ (Fig. 6, right). Using this value in equation 11 together with $K_{b}=91$ $\mathrm{N} / \mathrm{m}$ and $L_{b}=0.53 \mathrm{~m}$, the measured force at the frog in Fig. 4 can be well fitted with $T_{0}=50 \mathrm{~N}$ and $\alpha_{T}=8 \mathrm{~N} / \mathrm{N}$ (see Fig. 4 full lines)

Equation 11 gives an analytical relation between the transversal force at the frog and the bow force. This result will be used later to justify the way the force sensor will be calibrated and to interpret some of the observed results.

\section{DESIGN OF A BOW FORCE SENSOR}

A sensor for measuring the bow force in normal playing was designed, based on the principle described above by estimating the transversal force at the hair termination at the frog. The sensor was attached to the frog where the added weight gave minimum influence on the playing properties of the bow. The sensor was designed as a detachable unit, which could easily be moved to any bow without damaging the frog or hair.

\section{A. Description}

The force sensor consisted of a thin leaf spring of steel to which two strain gauges were glued (see Fig. 7). The steel strip was fixed to the flat side of the ferrrule by a clamping ring. The free end of the strip made contact with the bow hair through a light cylindrical bearing piece of wood. The bending of the strip generated a signal which after correction gave an accurate estimation of bow force. The strip was mounted on the lower side of the hair (the side facing the string), and a static down-ward bending was established as the hair was brought up to tension. When the bow was loaded in playing, the bending was reduced.

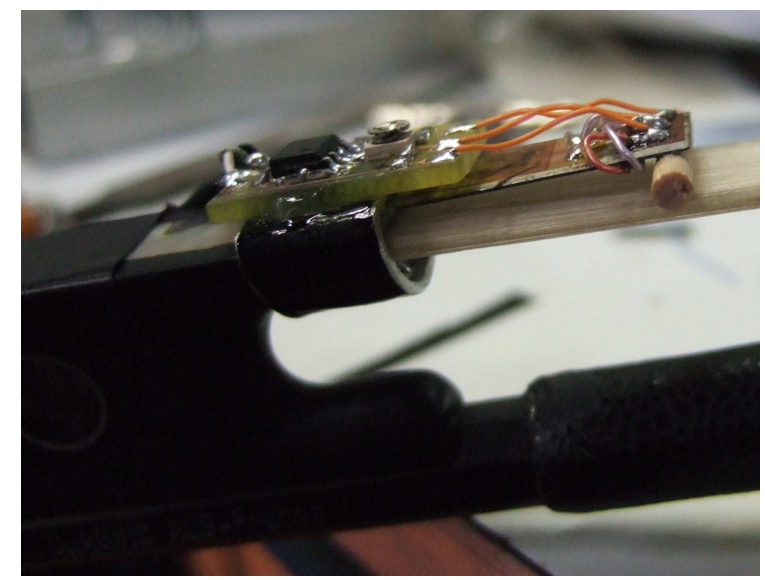

Figure 7. Bow force sensor. A thin steel strip is clamped to the flat side of the ferrule. The strip bends as the deflected bow hair presses at the free end via a wooden bearing piece. The bending is measured by two strain gauges glued to the strip. A Wheatstone bridge and conditioning amplifier is integrated in the sensor.

The strain gauges were glued to the upper and lower side of the strip and connected in one branch of a Wheatstone bridge (Vishay CEA series, 7 x $14 \mathrm{~mm}, 1000 \mathrm{ohm}$, gauge factor 2.1). Using this configuration, the output voltage will be linearly related to the resistance variations, and temperature changes will be compensated for. The resulting voltage variations were amplified using an operational instrumentation amplifier (gain 1000) integrated with the sensor. The electronics were connected to a battery and data aquisition board via a thin cable. The sensor was light, total mass $3.8 \mathrm{~g}$ including electronics and the clamping ring, which should be compared with the mass of the frog $(17 \mathrm{~g}$ including screw). The mass of the steel strip (0.6 g) and wooden bearing piece $(0.02 \mathrm{~g}$, diameter $2.5 \mathrm{~mm}$ ) was low compared to the bundle of bow hair (about $5 \mathrm{~g}$ ).

The strain $\epsilon$ and the deflection of the free end $\delta y$ of the steel strip when loaded by a transversal force $F_{L}$ at the free end is given by the equations for a bar with rectangular cross section clamped at one end 


$$
\epsilon=\frac{6 F_{L} b}{Y w t^{2}}=201 F_{L}[\mu \mathrm{m} / \mathrm{m}] \quad \delta y=\frac{4 F_{L} L^{3}}{Y w t^{3}}=0.14 F_{L}[\mathrm{~mm}]
$$

where $w=8.5 \mathrm{~mm}, L=20 \mathrm{~mm}$, and $t=0.5 \mathrm{~mm}$ are the width, length and thickness of the strip, $b=15 \mathrm{~mm}$ is the distance from the free end to the position where the strain is measured (center of the strain gauge, $b \leq L$ ), and $Y=210 \mathrm{GPa}$ is Young's modulus. The width of the strip is limited by the width of the bow hair, while $L, b$ and $t$ are used to obtain a suitable sensitivity. For practical reasons in playing, $L$ should not be longer than about $3 \mathrm{~cm}$. The diameter of the bearing piece $(2.5$ $\mathrm{mm}$ ) was set by sensitivity considerations (see Sect. III-B3).

The deflection of the steel strip in playing is small. For a typical bow force of $1 \mathrm{~N}$ at the middle of the bow, the transversal force on the strip $F_{L}$ can be estimated from Fig. 4 to be of about the same value, which deflects the free end around 0.1-0.2 mm. Even for very heavy loading with a bow force of about $1.5 \mathrm{~N}$ at the tip of the bow, the strip deflection will only be around $0.3 \mathrm{~mm}$. (Actually, the sensor operates in a reversed mode as mentioned, the deflection decreasing for increasing load.)

In order to check and calibrate the sensor, the bow was held as normal and pressed against a string-like facing ( $\mathrm{T}$ shaped piece, diameter $1 \mathrm{~mm}$ ) on a calibrated load cell. A comparison of the signals is shown in Fig. 8 . The responses are very similar with the exception of the smallest details, which have been smoothed slightly in the sensor signal.

An upper frequency limit for the dynamic response of the sensor was estimated from the resonance frequency, which was found experimentally to be around $400 \mathrm{~Hz}$.This was considered fully acceptable for the purpose of studying dynamical features of bow force related to the player's control of the bow.Vigorous spiccato playing on a completely rigid force transducer (BK8001, compliance $4 \cdot 10^{-6} \mathrm{~mm} / \mathrm{N}$ ) showed that the force signal did not include any significant components above $150-200$ $\mathrm{Hz}(-40 \mathrm{~dB})$. In real violin playing the compliant string will lower this frequency further.
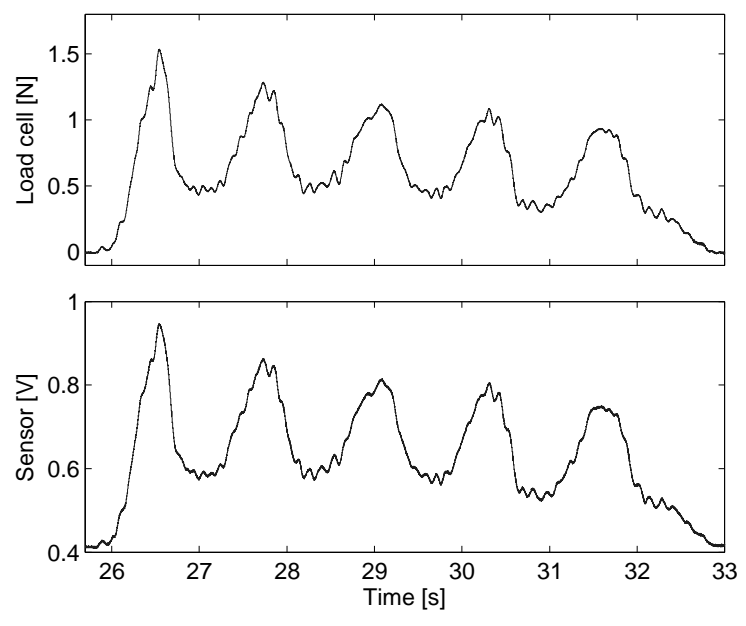

Figure 8. Illustration of the performance of the bow force sensor. The bow is repeatedly pressed against a calibrated load cell (top). The signal from the force sensor (bottom) replicates the force signal except for some minor high-frequency details.

\section{B. Design considerations}

1) Theoretical sensor sensitivity: The force that bends the strip is not identical to the transversal force at the frog $F_{1}$. The short distance $x$ between the frog and the bearing piece (where the bending force is applied), as well as the resulting deflection of the strip influence the estimation of $F_{1}$ and have to be taken into account.

In the following, the sensor is supposed to act on the bow hair as a simple spring $K$ at a distance $x$ from the frog and the bow hair is modelled as a single string with tension $T$. The rest position of the spring $y_{0}$ is set by the diameter of the bearing piece. Using this simple model described in Fig. 9, the corrected force $F_{L}$ acting at the free end of the strip can be computed as 
a)
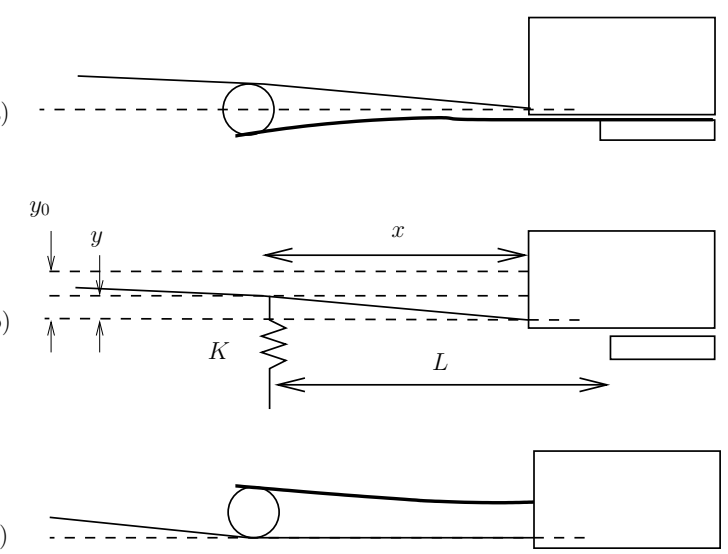

Figure 9. (a) Schematic view of the sensor. The steel strip experiences maximum deflection when the bow is unloaded. As bow force is increased the strip straightens. (b) Model used to derive the sensitivity. The strip is modeled as a spring (stiffness $K$, rest position $y_{0}$ ) acting on the bow hair at a distance $x$ from the frog. The bow hair is modelled as a single string with tension $T$. (c) Alternative design for minimizing $y_{0}$ with the strip mounted at the opposite of the bow hair.

$$
F_{L}=\frac{1}{T+K x}\left[-K x F_{1}+T K y_{0}\right]
$$

and, from Eq. 12, the deformation measured by the strain gauges can be expressed as

$$
\epsilon=\frac{3 b t}{2 L^{3}}\left[\frac{-x F_{1}+T y_{0}}{T+K x}\right] \quad K=\frac{Y w t^{3}}{4 L^{3}}
$$

It follows from Eq. 13 that the strain of the steel strip when placed under the bow hair is smaller than for the fixed-free condition, given by $\frac{3 b t}{2 K L^{3}} F_{1}$. Second, the strain is strongly non-linear as a function of $L$. For the free strip, the strain increases linearly with strip length (equation 12 with $b \propto L$ ), but the behaviour of the sensor strip under the bow hair is more complex.

In Eq. 14, the main design parameters $L, b, t, x$ and $K$ are constants. The tension $T$ is, however, set by the player and varies when a bow force is applied (up to $25 \%$ of the static tension, according to Sect. II). The transverse sensitivity $\partial \epsilon / \partial F_{1}$ of the sensor is given by

$$
\frac{\partial \epsilon}{\partial F_{1}}=\frac{3 b t}{2 L^{3}}\left[-\frac{x}{T+K x}+\frac{F_{1} x}{(T+K x)^{2}} \frac{\partial T}{\partial F_{1}}+\frac{K y_{0} x}{(T+K x)^{2}} \frac{\partial T}{\partial F_{1}}\right]
$$

Equation 15 gives the keys to the choice of design parameters for the sensor in order to reach maximal sensitivity and avoid strong non-linearity.

Focusing on the first term, the linearity of the response can be improved by increasing the stiffness $K$ which reduces the dependence on tension variations. If the actual tension is written $T=T_{0}+\delta T$ with $T_{0}$ the static tension set by the player, and if the maximal tension variation is $\delta T_{\max }$, a criterion for minimizing the influence of $\delta T$ is given by

$$
\frac{\delta T_{\max }}{T_{0}} \ll 1+\frac{K x}{T_{0}}
$$

A sufficiently high stiffness can consequently make the deformation practically independent of variations in bow hair tension and give the desired linear dependence of the transversal force at the frog. The influence of the static tension $T_{0}$ can be neglected provided that $K \gg T_{0} / x$, i.e. $K \gg 2500 \mathrm{~N} / \mathrm{m}$ with $x=20 \mathrm{~mm}$ and $T_{0}=50 \mathrm{~N}$. In the current design of the sensor $K$ is about $7000 \mathrm{~N} / \mathrm{m}$, and a maximal increase of the tension of $25 \%$ as observed in Fig. 6 would lower the sensitivity with about $6 \%$. Increasing the stiffness further reduces the strain and a compromise has to be found between linearity and sensitivity.

The second and the third term in Eq. 15 are directly dependent on the variations in bow hair tension. If the stiffness is high enough, the second term can be neglected. In the third term, the diameter of the bearing piece will influence the response 
through $y_{0}$. In order to reduce the non-linearity due to tension variations, it is desirable to make $y_{0}$ as small as possible, but that will reduce the maximum force that can be measured in the current configuration (strip mounted under the bow). An alternative design with the sensor mounted on the opposite side of the bow hair would minimize the influence of the third term (see Fig. 9, bottom).However, this configuration makes it necessary to fix the strip inside the frog, which is more radical operation when attaching the sensor to the ferrule.

2) Strip length: For the fixed-free strip, the strain $\epsilon$ increases with the strip length. In contrast, the sensitivity of the sensor, given in Eq. 15, shows a different behaviour and, under some conditions, increasing strip length can surprisingly reduce the sensitivity.

If tension variations are neglected in Eq. 15, the maximal sensitivity at $b=L$ is written as

$$
S=\frac{\partial \epsilon_{\max }}{\partial F_{1}}=\frac{3 t}{2 L^{2}} \frac{x}{T_{0}+K x}
$$

Fig. 10 illustrates the sensitivity given in Eq. 17 for a strip length $L$ varying from 0 to $5 \mathrm{~cm}$ and $x=L$ (full straight line). For comparison, the sensitivity of the fixed-free strip is shown (dashed line), varying linearly with the plate length. Between 0 and $2 \mathrm{~cm}$, the sensitivity of the sensor increases somewhat slower than the sensitivity of the fixed-free strip. Then it reaches a maximum value and slowly decreases from 3 to $5 \mathrm{~cm}$. In this region, the sensitivity is consequently reduced with increasing strip length.

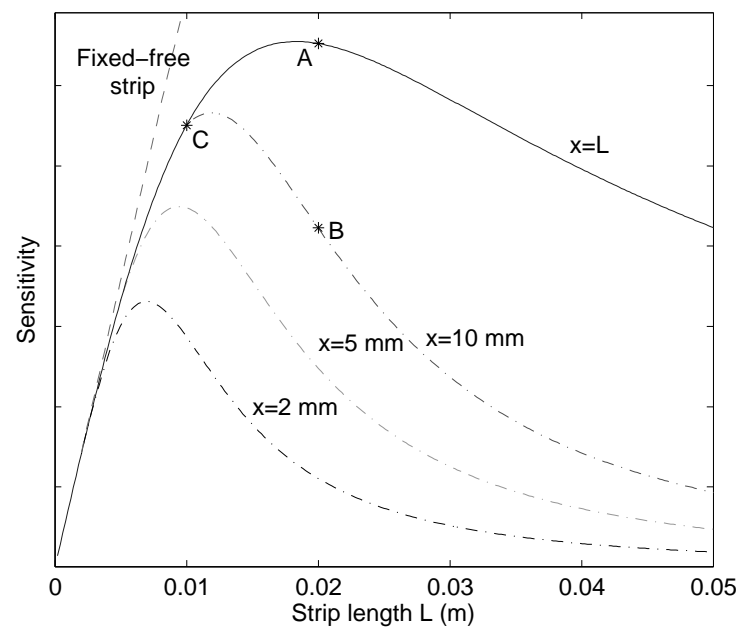

Figure 10. Variation of the sensor sensitivity with strip length $\mathrm{L}$ (full line, $x=L$ ). Other parameters are $\mathrm{Y}=2.1 \mathrm{MPa}(\mathrm{steel}), \mathrm{w}=5 \mathrm{~mm}, \mathrm{t}=0.4 \mathrm{~mm}$ and $T_{0}=50 \mathrm{~N}$. For comparison, the sensitivity of the fixed-free strip is shown (dashed line), as well as alternative sensor designs with $x<L$ (dashdot lines, marked $x=2, x=5$ and $x=10 \mathrm{~mm}$ ).

The fixation of the strip can be moved back on the ferrule in order to decrease the distance $x$ between the outer edge of the sensor and the frog, while keeping the same strip length and the same stiffness (see Fig. 9b). The idea of such a modification was to improve the playability (by decreasing the unused part of the bow hair) without modifying the mechanical response of the sensor. However, it can be seen in Fig. 10 that the sensitivity is significantly reduced. For example, with $L=20 \mathrm{~mm}$, the sensitivity decreases from A $(x=20 \mathrm{~mm})$ to $\mathrm{B}(x=10 \mathrm{~mm})$. Surprisingly, the response is even lower than the response of a strip with length $10 \mathrm{~mm}$ (point $\mathrm{C}$ ).

3) Bearing piece: As shown above the diameter of the cylindrical bearing piece should be as small as possible in order to minimize the non-linearity, but on the other hand it must at least touch the bow hair at the highest applied bow force. The diameter and position $(2.5 \mathrm{~mm}$ at $x=20 \mathrm{~mm}$ from the ferrule) was empirically determined by observing the displacement of bow hair for a realistic maximal bowing force near the frog.

In order be sure that the bearing piece stayed in place even in vigorous playing, it was glued to the strip in the initial experiments. However, this step of precaution introduced a hysteresis (see Fig. 11, right), resulting in higher sensor output 


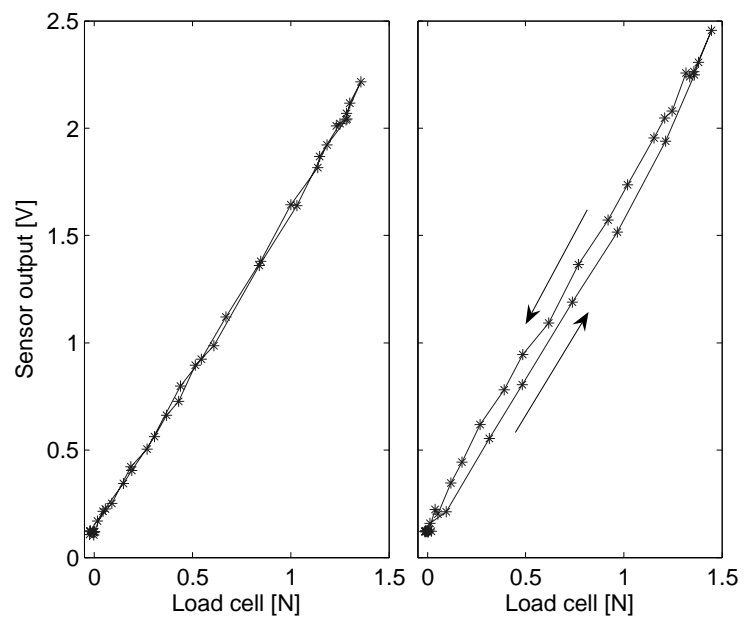

Figure 11. Effect of gluing the bearing piece to the strip. Left: Bearing piece wedged between the steel strip and the bow hair. Right: Bearing piece glued to the strip, resulting in hysteresis.

when the bow force was decreasing compared to when it was increasing. This effect could be related to a torque at the end of the strip. With the bearing piece glued, the contact with the strip is relatively strong. When the bow is pressed down, the hair stretches slightly, exerting a longitudinal force on the bearing piece in the direction towards the tip. The resulting couple on the strip counteracts the deformation due to the transversal force. When the bow force is reduced, the longitudinal force acts towards the frog, again counteracting the deformation change due to change in transversal force. When the bearing piece was held in position only by wedging between the strip and the bow hair, the hysteresis disappeared (see Fig. 11, left).

\section{CAlibration}

The sensor output reflects the transversal force exerted by the bow hair at the frog, which in turn depends on the bow force and bow position. The calibration procedure aimed at determining the coefficients that relate the sensor signal to the actual bow force. As the sensitivity was slightly dependent on the support of the bow, the calibration procedure was designed to be as close as possible to real playing conditions. This would also allow fast repeated calibrations during measuring sessions in order to take changes in hair tension into account.

\section{A. Calibration procedure}

The calibration was performed by pressing the bow against a load cell. The subject (violinist) was asked to hold the bow as in normal playing and apply equal bow force at successive bow positions, from the frog to the tip. The force signal from the load cell $F_{L C}$ which gave a calibrated reference of the bow force, was recorded together with sensor signal $s_{F}$. A typical calibration session is illustrated in Fig. 12.

In this example, ten successive bow positions were used, from the frog $\left(x_{\mathrm{b}}=35 \mathrm{~mm}\right)$ to the tip $\left(x_{\mathrm{b}}=610 \mathrm{~mm}\right)$. At each position, the bow force was varied periodically five times between about 1-2 N, each cycle lasting $1 \mathrm{~s}$ approximately. The variation range in bow force was approximately the same for the ten positions, but the signal from the force sensor decreased when approaching the tip due to the position of the sensor.

\section{B. Calibration coefficients}

The signals were segmented in order to get calibration data for each bow position $x_{b}$. Calibration curves were obtained by fitting a second order polynomial to the data

$$
s_{F}=b_{2}\left(x_{b}\right) F_{L C}^{2}+b_{1}\left(x_{b}\right) F_{L C}
$$



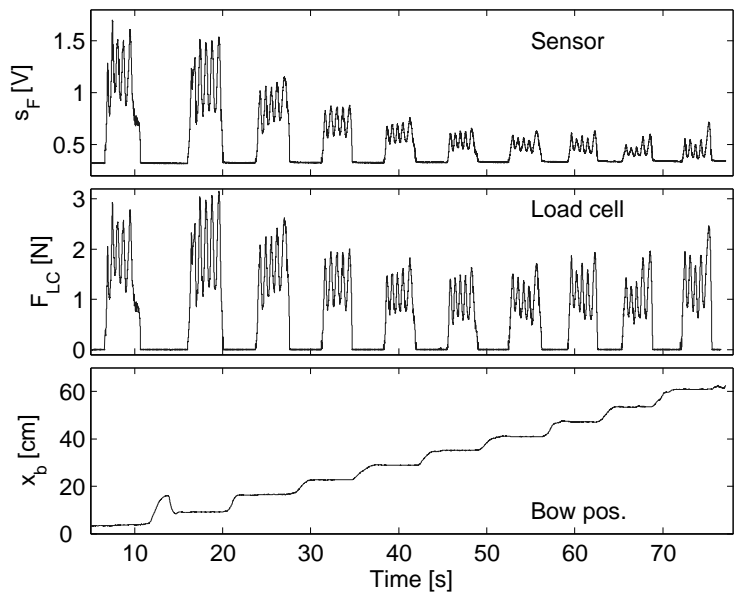

Figure 12. Calibration procedure. The bow is pressed against a calibrated load cell at 10 bow positions. At each position the bow force is varied periodically five times. Bow positon (bottom), bow force as measured by the load cell (middle), and force sensor signal (top).

Fig. 13 shows an example with three calibration curves for a violin bow, corresponding to bow positions at the frog, middle and tip.

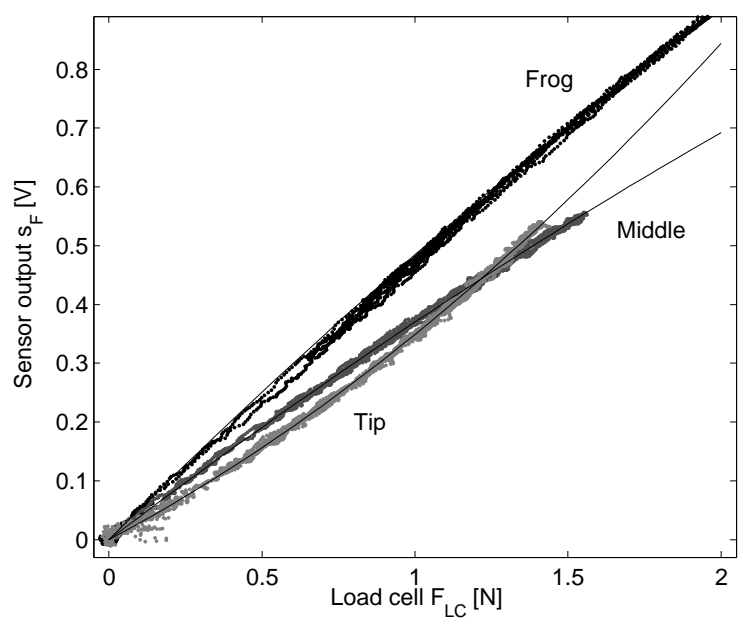

Figure 13. Calibration curves for a violin bow corresponding to bow positions near the frog, middle and tip. Fitted second-order polynomials are shown with solid lines.

Fig. 14 shows the fitted calibration coefficients $b_{1}$ and $b_{2}$ plotted as function of bow position for three repeated calibrations. The calibration procedure is seen to give good reproducibility. The figures suggest that $b_{1}$ can be well approximated by a linear fit, whereas $b_{2}$ requires quadratic interpolation.

The correspondence with the analytical expressions derived in Sect. III is good. From equation 11, with $\gamma=x_{b} / L_{b}$, it could be predicted that $b_{2}$ and $b_{1}$ should show the observed dependence on bow position

$$
\begin{gathered}
b_{2}\left(x_{b}\right) \propto x_{b}^{2} \frac{\alpha_{T}}{K_{b} L_{b}^{3}} \\
b_{1}\left(x_{b}\right) \propto 1-\frac{x_{b}}{L_{b}}\left(1-\frac{T_{0}}{K_{b} L_{b}}\right)
\end{gathered}
$$



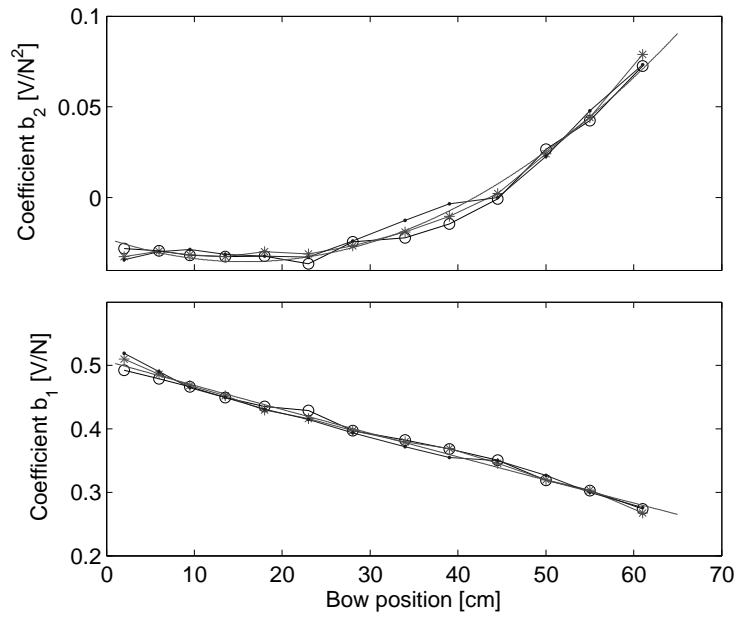

Figure 14. Calibration coefficients $b_{2}$ and $b_{1}$ plotted as function of bow position $x_{b}$ for three repeated calibrations.

\section{Bow force reconstruction}

During measurements, bow force is calculated from the sensor signal and bow position. Using equation 18, the actual bow force $F$ can be reconstructed as

$$
F=\frac{-b_{1}\left(x_{b}\right)+\sqrt{b_{1}\left(x_{b}\right)^{2}+4 b_{2}\left(x_{b}\right) s_{F}}}{2 b_{2}\left(x_{b}\right)}
$$

Of the two possible solutions to Eq. 15 this was chosen because it has the necessary properties for the reconstruction: When $b_{1}$ is positive (which is always the case), $F$ will be zero when $s_{F}=0$. Moreover, whatever the sign of $b_{2}$, this function is increasing when $s_{F}$ increases.

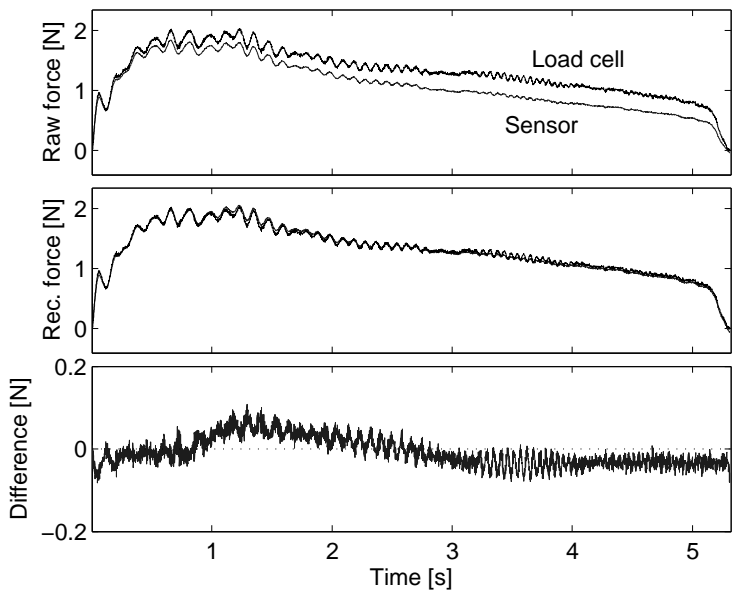

Figure 15. Test of the reconstructed bow force during a full down-bow from frog to tip. The bow was moved with constant velocity on a wheel mounted on the load cell. Bow position was interpolated linearly between the beginning and end of stroke. Top: Comparison between load cell output and raw sensor signal. The sensor signal has been normalized using $b_{1}$ and $b_{2}$ at the frog. Middle: Comparison between the load cell signal and reconstructed bow force. Bottom: Difference between the reconstructed bow force and load cell output.

In order to check the reconstruction using interpolated calibration coefficients $b_{2}\left(x_{b}\right)$ and $b_{1}\left(x_{b}\right)$, the bow was 'played' on a wheel mounted on the calibrated load cell, using full bow strokes with constant velocity (see Fig. 15). The load cell signal and the reconstructed bow force are seen to almost coincide, the difference being only $\pm 0.05 \mathrm{~N}$ centered around zero. This value can be considered as a typical error in the bow force estimation under standard conditions (slow variations of the bow force between 0.5 and $2 \mathrm{~N}$ ). 


\section{Factors influencing the calibration}

The output of the bow force sensor is sensitive to several conditions, and it is not straight-forward to estimate the effect and compensate for the effect of all of them. In this section, three factors are discussed, including the mechanical action of the bow, hair tension, and tilting of the bow, and their influence on the calibration coefficients illustrated.

1) Influence of the mechanical action of the bow: More than 20 bows were tested with the sensor, from violin bows to double bass bows. Most of them showed the characteristics shown in Fig. 13: The calibration curves for different bow positions lie rather close together, and the curve for the tip steepens rapidly for higher bow forces. Further, the output from the sensor is typically higher for bow positions at the tip than at the middle of the bow. Some bows, however, showed a different behaviour as illustrated in Fig. 16 where the bow in Fig. 13 is compared with a bow which behaves quite differently. Here the calibration curves are widely separated, and the response close to the tip is weak for bow forces below $1 \mathrm{~N}$. For some very stiff bows, like double bass bows, this behaviour is easily understandable, as the bow can be represented by the rigid model described in Sect. II. For violin bows, however, the comparison between calibration curves and measurements of the transversal stiffness of the bow did not show a specific relationship. In these cases, the weak response below $1 \mathrm{~N}$ seems to be related to some more elaborated property of the bow.
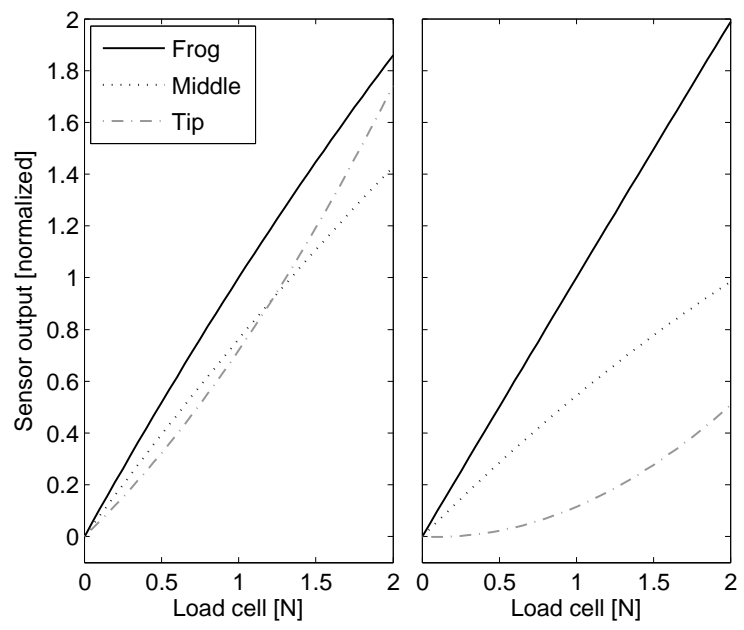

Figure 16. Calibration curves for two violin bows with different mechanical action. Left: Bow 1, corresponding to Figs. 13 and 14 . Right: Bow 2, with a stiffer stick of carbon fibre which gives widely separated calibration curves with a much lower sensitivity at the tip than at the frog.The curves have been normalized using the sensor output for $1 \mathrm{~N}$ force at $5 \mathrm{~cm}$ from the frog as reference.

The calibration procedure will take such differences in mechanical action between bows into account, but the accuracy in the reconstruction of low bow forces will be reduced. For example, at bow forces between 0.5 and $1 \mathrm{~N}$, a measurement error in the sensor output of $1 \%$ would give an error of about $3 \%$ in the reconstructed bow force for bow 1, but as high as $15 \%$ for bow 2. At $1.5 \mathrm{~N}$ the errors have reduced to $1.8 \%$ for bow 2 and $0.7 \%$ for bow 1 . In short, some bows will be more suitable for precise bow force measurements with this design of the sensor than others because of their mechanical properties.

2) Influence of bow hair tension: The tension of bow hair set by the player influences the transversal force at the frog (see Section II) and the response of the sensor (see Section III). The combined effect of changes in hair tension was quantified in a calibration with three largely deviating tensions; very low, normal, and very high, the two extreme values not being suitable for playing. The results are shown in Fig. 17.

The results for bow positions at the frog follow the expected behaviour. Close to the frog the stick does not bend and tension can be supposed to be constant during loading $\left(T=T_{0}\right.$ ). Equations 11 and 14 , with $\gamma \rightarrow 0$, predict that $b_{1}$ will be inversely proportional to $T_{0}$, which is in line with the observations.

An interpretation of the results close the tip would require knowledge about the mechanical behaviour of the particular bow. Here it is sufficient to note that the values of calibration coefficient $b_{1}$ for different tensions merge when approaching the tip. 


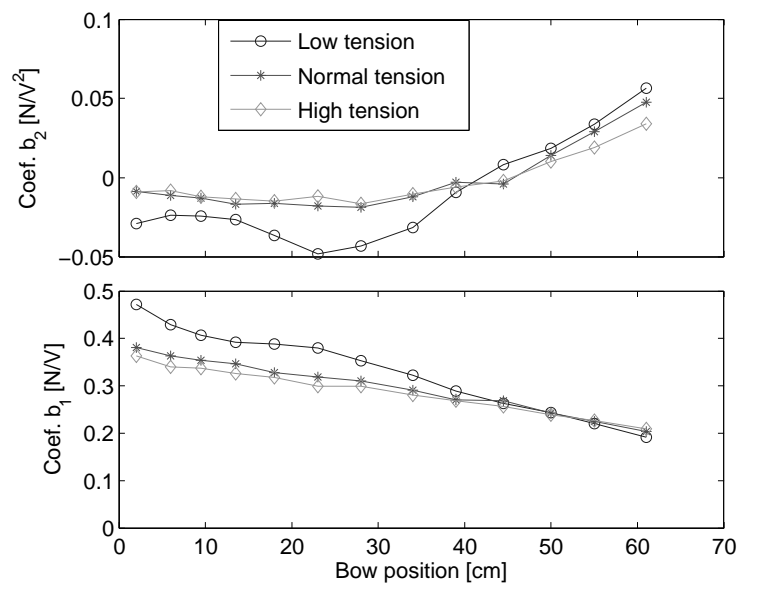

Figure 17. Influence of the bow hair tension on the calibration coefficients for three values; low, normal, high. The high and low tensions were not suitable for playing.

The observed changes in $b_{2}$ and $b_{1}$ are seen to be rather limited, even for extreme values of tension. Typical differences in preferred tensions between players are much smaller and will be handled accurately by the calibration procedure. However, repeated calibrations during a measurement session are desirable in order to take natural changes in hair tension during playing into account.

3) Influence of bow tilt: String players tilt the bow in playing in order to reduce the effective width of the bow hair in contact with the string. Typically the bow is tilted much in soft playing, and more so when playing near the frog than at the tip. While the mechanical characteristics (stiffness) of the bow as well as changes in tension are taken into account by the calibration as discussed above, bow tilt is an external parameter which not easily can be handled in the measurements.

The tilting influences the bow force measurement in two ways. First, the sensor measures the force applied in the direction perpendicular to the steel strip, whereas the bow force by definition is applied perpendicular to the string. This results in an underestimation of the bow force, related to the tilt angle. For instance, an angle of $20^{\circ}$ would give $6 \%$ lower transversal force acting on the sensor (proportional to $\cos \theta_{\text {tilt }}$ ), compared to the case with the bow hair flat on the string.

Secondly, the bending of the strip, and thus the response of the sensor, will be affected when the transversal force from the hair is not evenly applied across the bearing piece. In addition to bending the strip will twist. The profile of the transversal deflection of the bow hair as it crosses the bearing piece is not easily predicted. However, as the ribbon of bow hair is composed of several layers, a force which is applied mainly at one edge of the ribbon will be distributed approximately linearly across the width of the bearing piece (see discussion in [8][9])

The uncertainties in the estimation of bow force due to tilting were found to be limited. Calibrations performed when tilting the bow at various degrees showed that the influence was noticeable only for bow positions close to the sensor. The deviation between the calibration curves with and without tilting the bow could then reach more than $30 \%$. From the second calibration point at about $8 \mathrm{~cm}$ from the frog and further towards the tip the deviation was found to be less than $10 \%$. In order to measure bow force correctly also in tilting, a possible solution would be to split the strip along the centerline and apply two sets of strain gauges, one on each side. In this way the amount of tilting could be estimated and a correction for the influence on bow force taken into account.

\section{APPLICATIONS IN PLAYING}

The use of the bow force sensor is illustrated in two applications. In the first example, one sensor was placed at the frog as described above, and motion capture technique [10][12] was used to measure bow position in order to reconstruct the bow force. Alternative methods for measuring bow position include a simple solution using a resistive wire placed among the bow hairs and connected in a Wheatstone bridge [1], [2], and more complex systems based on a capacitive principle with an antenna 
placed behind the bridge of the violin and a resistive strip along the bow stick [13]. In the second application example, two sensors are used, one at the frog and one at the tip. In this configuration, the difference between the sensor signals could be used to calculate bow position and no extra measurement devices were needed.

In the experiments two violinists were asked to play two types of bow strokes; sustained, separated notes (detaché) in which the bow force was supposed to be held rather constant, and long strokes with strong accents at the beginning of each note, which would require large variation in bow force.

\section{A. One bow force sensor and motion capture}

Examples using one bow force sensor and motion capture are given in Fig. 18, showing audio signal, bow force, bow position and bow velocity, for a G4 note played without vibrato on the D string. In Fig. 18a showing detaché , it is apparent from the audio signal that each note was played with a slight crescendo. This was accomplished by an increase in bow velocity from about 30 to $60 \mathrm{~cm} / \mathrm{s}$ during each stroke. The reconstructed bow force was approximately constant at about $600 \mathrm{mN}$, except local increases prior to the bow changes at the frog. The raw force sensor signal, which decreased as expected when approaching the tip, illustrates the magnitude of the compensation for bow position in the reconstruction of bow force.
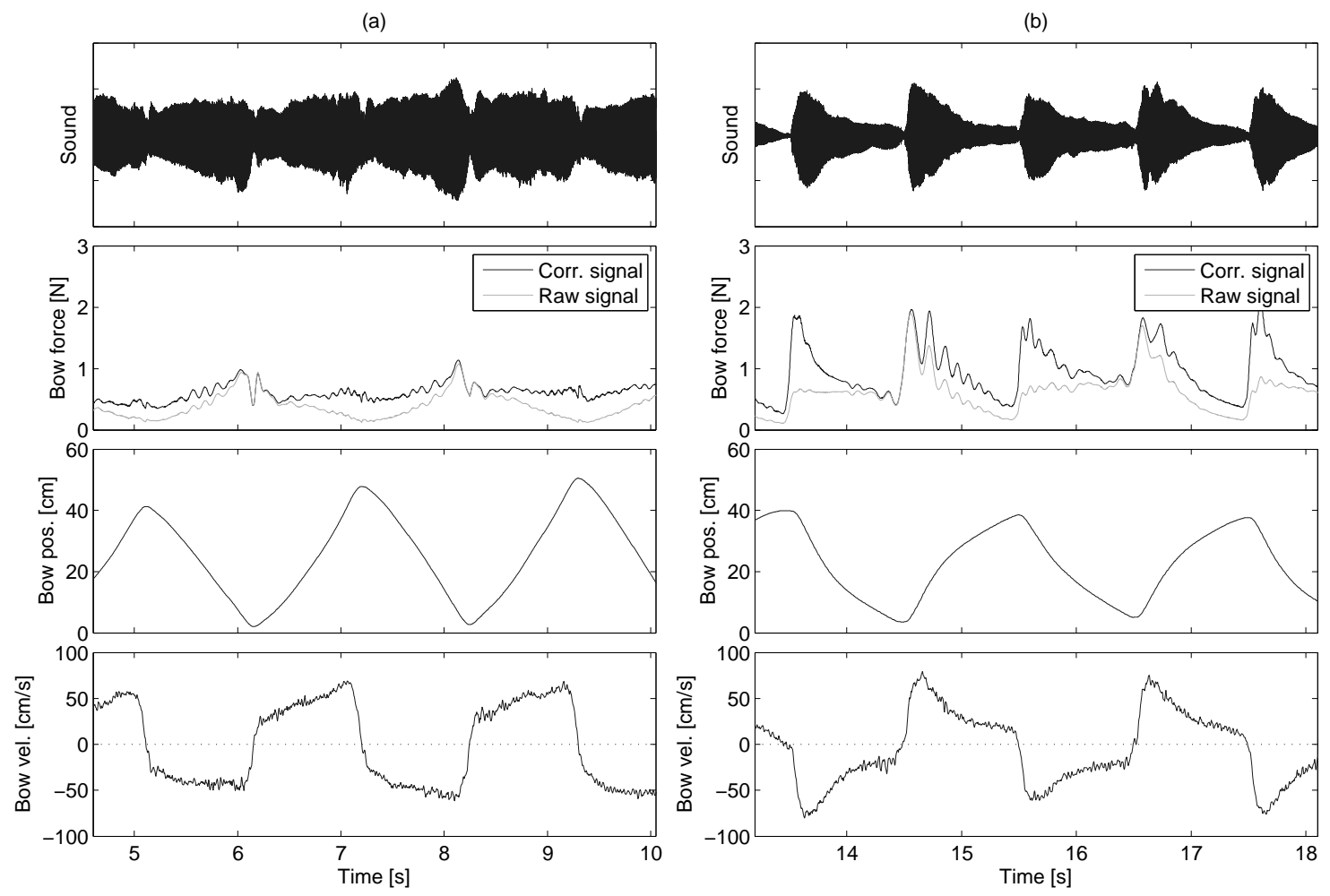

Figure 18. Measurements using one bow force sensor and motion capture showing audio signal, reconstructed bow force, bow position and bow velocity (top to bottom). The raw sensor signal is included in the bow force panel (gray curve) in order to illustrate the effect of bow position on the reconstruction. Bow velocity was derived from the bow position signal. The player performed (a) sustained, separated notes (detaché), and (b) long strokes with inital accents on each note.

The compensation is more promounced in Fig 18b showing the bow strokes with accents: In up-bows (negative velocity), the raw sensor signal is almost flat, whereas the reconstructed bow force shows the same large variation as in down-bows. The accented initial part of each note is obtained through a coordination of high bow velocity reaching about $80 \mathrm{~cm} / \mathrm{s}$ and high bow force, up to $2 \mathrm{~N}$. The sustained softer parts are played with a comfortable bow velocity around $20 \mathrm{~cm} / \mathrm{s}$, requiring about $0.5 \mathrm{~N}$ bow force. 


\section{B. Two bow force sensors}

An alternative system was developed in order to allow bow force measurements without an additional device for measuring bow position. The solution was based on a second force sensor at the tip. This configuration was originally used by Askenfelt [1], [2], but he used the two sensors (four strain gauges) together in a full Wheatstone bridge for bow force. The sensitivities of the frog and tip branches were adjusted to obtain a constant signal for a given bow force at all positions along the bow hair.

In the current application the two sensors were used independently to obtain the transversal force at the frog and tip, respectively. When a certain bow force is applied somewhere along the bow, the outputs from the sensors will be dependent on the actual bow position. The difference between the signals can be used to estimate the bow position. By simultaneous calibration of bow force and bow position it is thus possible to obtain both parameters from the output of the two force sensors.

The second sensor was clamped between the bow hair and the ivory plate at the tip. A thin metal fixation around the head prevented the sensor from moving.

1) Calibration: A coordinated calibration of bow force and bow position was performed by pressing the bow hair against the load cell at different bow positions as described in Sect. IV. Signals obtained from the sensor at the frog $\left(F_{c 1}\right)$ and at the tip $\left(F_{c 2}\right)$ were used to compile a calibration grid from which bow force and bow position could be interpolated (see Fig. 19, left). The grid gives the bow force and bow position for equally spaced values of $F_{c 1}$ and $F_{c 2}$. An analytical expression as for the case with one force sensor (see Eq. 21) was not considered due to the complex relation between the rotation of the head and the output of the sensor at the tip.

(a)

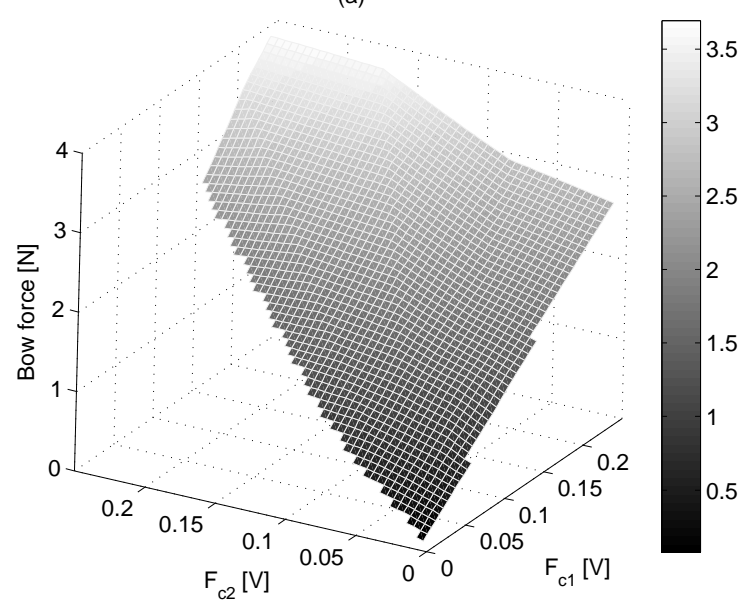

(b)

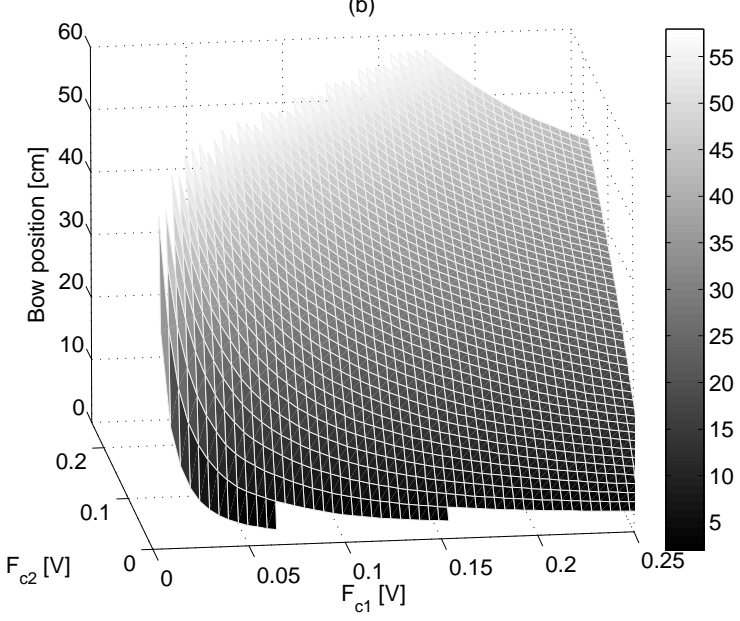

Figure 19. Coordinated calibration surfaces for (a) bow force and (b) bow position using one sensor at the frog $F_{c 1}$ and one at the tip $F_{c 2}$.

As seen in Fig. 19a, the force calibration surface is delimitated by two edges originating from the origo of $F_{c 1}$ and $F_{c 2}$, the right-going corresponding to a bow position at the frog and the left-going to a position near the tip. Near the frog, the variations in $F_{c 1}$ are large as the bow force is varied, whereas the tip sensor provides a signal $F_{c 2}$ with very small variations. However, near the tip, $F_{c 2}$ variations are much stronger, and $F_{c 1}$ still has appreciable variations. This is in agreement with the observations in the previous sections, showing that the frog signal response does not tend to zero when bowing near the tip. A similar calibration grid was computed for bow position, giving bow position as a function of $F_{c 1}$ and $F_{c 2}$ (see Fig. 19b).

Recordings of the two sensor signals were used to determine bow force and bow position from two-dimensional interpolations on the calibration surfaces. It should be noted that as the slope of the bow position surface was very steep near the origin, bow position determination can be supposed to work better for high bow forces than for lower.

2) Measurements: As before, a violinist was asked to play sustained bow strokes with and without accents. The examples were played on the open $\mathrm{G}$ string, and the bridge force signal was recorded by a transducer on the bridge. Bow position was computed from the sensor signals and calibration grid. The resulting bow position signal was rather noisy, and a Savitzky-Golay filter (polynomial order 2, frame size 61) was used for smoothing before calculating the bow velocity. 
(a)
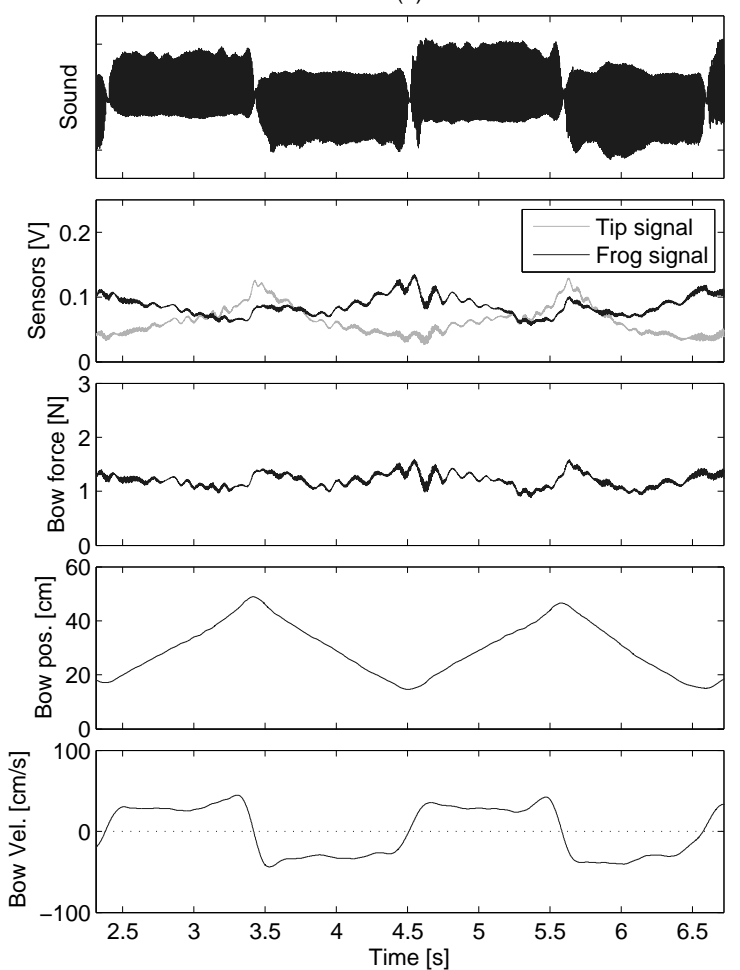

(b)
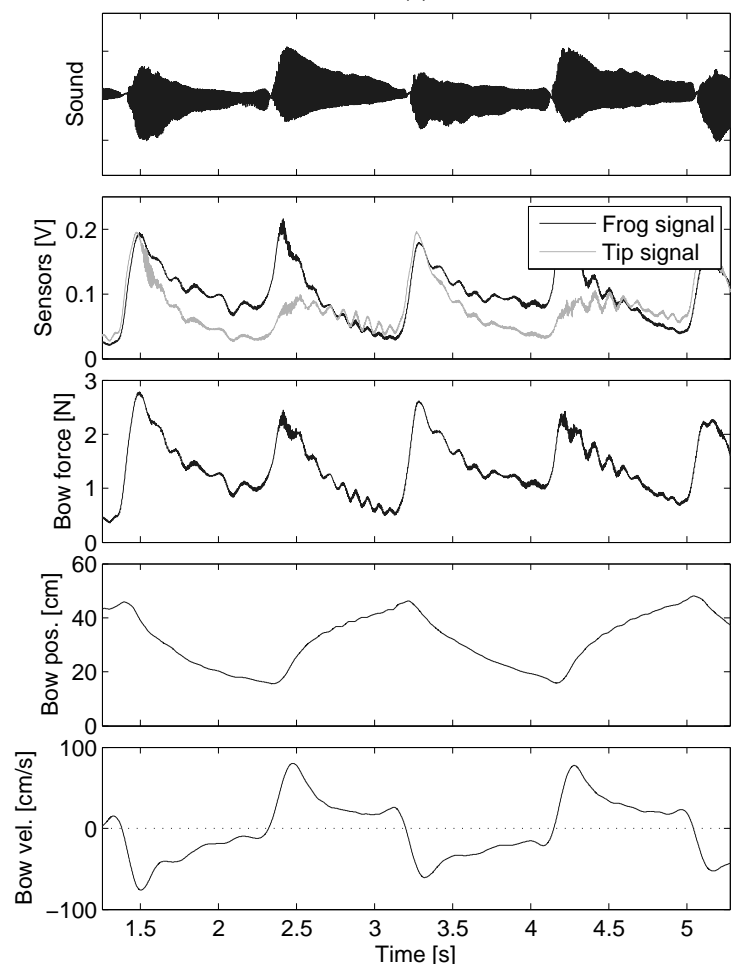

Figure 20. Measurements using two force sensors, one at the frog and one at the tip, showing bridge force, output from the two force sensors (frog, black line; tip, grey line), interpolated bow force, bow position and bow velocity. Bow velocity was derived from the bow position signal. The player performed (a) sustained, separated strokes (detache'), and (b) long strokes with initial accents on each note.

The results are shown in Fig. 20. It is satisfying to notice the good agreement in bow position and velocity with the corresponding curves in Fig 18, obtained by motion capture. The bow position curves are almost triangular for the detaché notes, whereas the accentuated examples show asymmetrical patterns due to the high velocity during the attack of the note. The bow velocity curves show very convincing similarities, including the peak velocities around $80 \mathrm{~cm} / \mathrm{s}$. Further, the velocity peaks are well correlated with the bow force peaks. In both cases, each maximum in bow force occurs just before the corresponding velocity maximum, the shift being between 0 and 100 ms (cf. Figs. 18 and 20).

In the detaché example (see Fig. 20a), the bow velocity was lower than in the previous example (see Fig. 18a), about 30 $\mathrm{cm} / \mathrm{s}$ and with a short period of acceleration before the bow changes at the tip. The bow force was almost constant as in Fig 18 but higher, above $1 \mathrm{~N}$. The two raw sensors signals are shown separately for comparison. The signal variations are seen to be in opposite phase; the tip signal increases and the frog signal decreases in down-bows (positive velocity), and inversely in up-bows. After the reconstruction, the interpolated bow force signal is rather flat, with a mean value around $1.2 \mathrm{~N}$.

In the accentuated example (see Fig. 20b), both bow velocity and bow force are of similar magnitude as in Fig. 18b. As the bow force was varied, the two sensor signals followed each other close in phase. It could be noted that the tip signal is much stronger when the attack is played close to the tip in up-bows (negative velocities), whereas the signal from the frog sensor has almost the same amplitude in accents played at the tip as at the frog. Again, this is related to the fact that the response of the frog sensor does not go down to zero when playing near the tip.

The comparisons between the examples measured with the motion capture (Fig. 18) and two force sensors (Fig. 20) suggest that the configuration with two force sensors offers a satisfying alternative for measuring bow position as well as bow force. In many cases there is thus no need for dedicated extra equipment for position measurement. 


\section{SUMMARY AND CONCLUSIONS}

The principle and implementation of a sensor that can be used to measure the bow force during violinists' performances are presented. The sensor is based on measurement of the transversal forces at the bow hair terminations when the bow is pressed against the string. The principle was tested in laboratory experiments, in order to verify the influence of bow position and hair tension on the forces at the terminations. Somewhat unexpected, it was found that the transversal force at the frog termination could increase when the loading point on the bow hair was moved closer to the tip. By measurements of variations in bow hair tension and the displacement of the tip due to stick bending, a simple model describing the mechanical behaviour of the bow could be developed, which predicted the measured force at the frog well.

The design of the sensor was described in detail. The sensor is light (total mass $<4 \mathrm{~g}$ ) and easily detachable, causing no damage to the bow. It consists of a thin leaf spring of steel that is fixed at the ferrule and contacts the bow hair through a light bearing piece. The steel strip is deformed due to the hair deflection, and the deformation is measured using strain gauges glued on both sides of the strip.

A procedure for calibrating the sensor under conditions resembling normal playing was described. The player presses the bow against a calibrated force transducer at successive positions along the bow, and varies the bow force periodically. Two calibration coefficients were derived that are used to reconstruct the bow force from the sensor signal and the bow position. The reconstructed bow force was found to be accurate. The typical error in bow force estimation under standard conditions (slow variations between 0.5 and $2 \mathrm{~N}$ ) was about $0.05 \mathrm{~N}$. The influence of the mechanical properties of the bow, hair tension, and tilting were illustrated and quantified.

Measurements of bow force in normal violin playing were illustrated for two applications of the sensor. The first used only one sensor at the frog and requires another device for measuring the bow position in order to reconstruct the bow force. In the second application two sensors were used, one at the frog and one at the tip, in order to measure both bow force and bow position. The bow force sensor was found to perform satisfactorily in both applications, giving realistic measurements.

In summary, the described bow force sensor is a robust and accurate device for simple measurements of bow force in string playing using the player's own bow, and without interfering with normal playing conditions.

\section{ACKNOWLEDGEMENTS}

This work is part of the CONSONNES project, funded by the French Agence Nationale pour le Recherche. Stays at IDMIL, McGill University, Montreal, and at the Dept. of Speech, Music and Hearing, Royal Institute of Technology (KTH), Stockholm, were funded by the Cost 287-ConGas action and the Swedish Institute. Special thanks go to Emmanuel Fléty and Alain Terrier for their valuable help in developing the sensors and very special thanks go to Benoît Fabre for his comments and corrections during the writing of this manuscript.

\section{REFERENCES}

[1] A. Askenfelt. Measurement of bow motion and bow force in violin playing. J. Acoust. Soc. Am., 80(4):1007-1015, 1986.

[2] A. Askenfelt. Measurement of the bowing parameters in violin playing II. J. Acoust. Soc. Am., 86(2):503-516, 1989.

[3] A. Askenfelt. Observations on the dynamic properties of bows. Speech Transmission Laboratory Quarterly Progress and Status Report, Dept. of Speech, Music and Hearing, Royal Institute of Technology (KTH) Stockholm, STL-QPSR, 4:43-49, 1992.

[4] M. Demoucron, A. Askenfelt, and R. Caussé. Mesure de la pression d'archet des instruments à cordes frottées, application à la synthèse sonore. In 8ème Congrès Français d'acoustique, Tours, France, 2006.

[5] E. Guaus, J. Bonada, A. Perez, E. Maestre, and M. Blaauw. Measuring the bow pressing force in a real violin performance. In Proceedings of International Symposium on Musical Acoustics, Barcelona, Spain, 2007.

[6] K. Guettler. On the creation of the Helmholtz movement in the bowed string. Acta Acustica, 88(6):970-985, 2002.

[7] K. Guettler, E. Schoonderwaldt, and A. Askenfelt. Bow speed or bowing position - which one influences spectrum the most? In Proceedings of the Stockholm Music Acoustics Conference (SMAC 03), pages 67-70, Stockholm, August 2003.

[8] R. Pitteroff. Contact mechanics of the bowed string. PhD thesis, University of Cambridge, 1995.

[9] R. Pitteroff and J. Woodhouse. Mechanics of the contact area between a violin bow and a string. Part III: Parameter dependence. Acta Acustica, 84:929-938, 1998.

[10] N. Rasamimanana, D. Bernardin, M. Wanderley, and F. Bevilacqua. String bowing gestures at varying bow stroke frequencies: A case study. In Gesture Workshop, Lisbon, Portugal, 2007. 
[11] J. C. Schelleng. The bowed string and the player. J. Acoust. Soc. Am., 53(1):26-41, 1973.

[12] E. Schoonderwaldt, S. Sinclair, and M. Wanderley. Why do we need 5-DOF force feedback? An analysis of violin bowing. In 4th International Conference on Enactive Interfaces (ENACTIVE07), Grenoble, France, 2007.

[13] D. Young. the hyperbow controller: real-time dynamics measurement of violin performance. In Proc. of the 2002 Conference on New Instruments for Musical Expression (NIME-02), Dublin, Ireland, 2002. 